\title{
Why ICD still send out atrial pacing pulse after ventricular extrasystole occurrence in a patient with old myocardial infarction
}

\author{
Kang $\operatorname{Li}^{1}$, Yansheng Ding ${ }^{1}$, and Jing Zhou ${ }^{1}$ \\ ${ }^{1}$ Peking University First Hospital
}

June 1,2020

\section{Why ICD still send out atrial pacing pulse after ventricular extrasystole occurrence in a patient with old myocardial infarction}

Kang Li,MD,Yansheng Ding,MD ,Jing Zhou*,MD

Department of Cardiology, Peking University First Hospital, Beijing, China

*Corresponding author: Jing Zhou

zhoujing123@gmail.com

Postal address:

Xishiku Str.8,Xicheng District,Beijing,China,100034

Conflict of interest:None

A 78-year-old male with ischemic cardiomyopathy and old myocardial infarction (the posterior inferior left ventricle and interventricular septum), left ventricular ejection fraction $30 \%$,initially had a dual-chamber implantable cardioverter-defibrillator (ICD) placed 6 years ago. After that, AAI pacing was dominant in managed ventricular pacing mode (MVP mode :AAIR $<=>$ DDDR and AAI $<=>$ DDD,Medtronic), the pacing rate was previously set to 55 times / $\mathrm{min}$ ), with paroxysmal atrial fibrillation and frequent premature ventricular contraction (PVC), without sustained ventricular tachycardia recording or discharge events. And judging from QRS morphology of the PVC: it maybe originated from the scar zone of old myocardial infarction in the posterior inferior left ventricle.(Figure-1:)

He underwent a routine ICD (Medtronic Evera S DR DDBC3D4) replacement when the existing device reached the recommended replacement time. Both leads were tested at that time and found to be fine,located in the right auricle and the right ventricular apex. Just after the replacement procedure,we found something like troubleshooting(Figure-1: ).

Why did ICD still send out atrial pacing(AP) pulse and follow-up ventricular safety pacing (VSP) after the occurrence of PVC? Obviously,there was a significant delay from the onset of PVC to the sense of PVC by the ventricular electrode(VS). The interval between PVC and previous AP was $920 \mathrm{~ms}$, and the right ventricular electrode was delayed for $90 \mathrm{~ms}$ to detect PVC(Figure-1: ). Because of the default pacing rate of 60 times / min, ICD sended out AP pulse according to the pacing interval of $1000 \mathrm{~ms}$, which was $10 \mathrm{~ms}$ earlier than the delayed perception of PVC. Then the ventricular electrode sensed the ventricular activation of PVC,and sended out the VSP after the AP pulse(Figure-1:). When VSP function was turned off, ICD still sended out AP pulse after PVC as a result of the conduction delay of 90ms, but no VSP was released again. AP produced atrial activation and failed to transmit down due to falling into ventricular refractory period(Figure-1:). 
We adjusted the pacing rate of ICD from 60 to 55 times / min, then the pacing interval was $1090 \mathrm{~ms}$. The ventricular electrode could sense the $\mathrm{PVC}(920 \mathrm{~ms}+90 \mathrm{~ms}=1010 \mathrm{~ms})$ from the delayed conduction to the left ventricular posterior inferior origin without overlapping the AP pulse. After MVP turned on, it took 40 $\mathrm{ms}$ for $\mathrm{AP}+\mathrm{VS}$ to transmit the ventricular activity from the atrioventricular node to the right ventricular electrode, and $90 \mathrm{~ms}$ for AP+PVC to transmit the scar zone of ventricular myocardium due to old myocardial infarction to the right ventricular electrode(Figure-2:). We modified the sense polarity of the right ventricular electrode from "tip to ring" to "tip to coil", but there seemed to be no significant difference in the delay of ventricular perception.

Later, when the patient's oral dosage of beta blocker was increased, the AV delay was prolonged and the conduction block was more severe than before. Because of MVP function, inhibiting ventricular pace(VP), thus $\mathrm{AP}+\mathrm{VSP}$ and AS without VP appeared regularly (Figure-2: ). Because of the regular occurrence of ventricular pacing missing, the patient's palpitation became worse.And the increase in the proportion of right ventricular apical pacing may worsen cardiac function. Finally, our solution is to reduce the dosage of beta blocker, set ICD pacing rate of 55 times / min, MVP on, VSP on, and suggested the strategy of catheter ablation of PVC.

The main reason for the delay of ventricular electrode perception is the slow conduction through the scar zone of posterior inferior left ventricle and interventricular septum due to the old myocardial infarction(Figure3 ). The second reason is the deterioration of conduction system itself(senile degeneration, old myocardial infarction involving conduction system, ventricular septal conduction time delay). The latter was confirmed by the significant atrioventricular block after increasing the dose of beta blocker. 


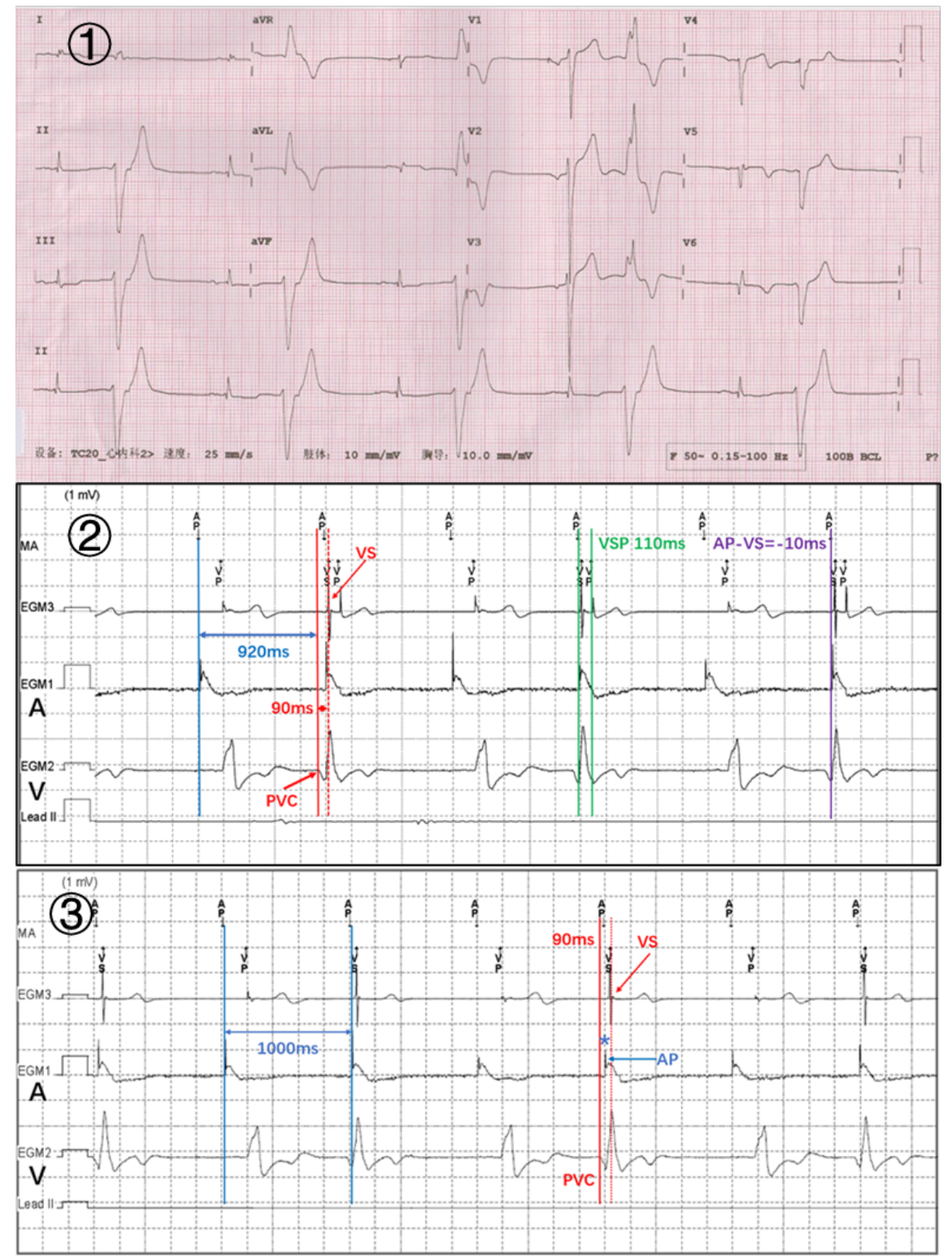

Figure-1:AAI pacing mode and frequent premature ventricular contraction(PVC). Judging from QRS morphology of the PVC: it originated from the scar zone of old myocardial infarction in the posterior inferior left ventricle.AP+VSP overlapped in PVC?AP overlapped in PVC when VSP function turned off. EGM 1:Atip to Aring;EGM 2:Can to RV coil;EGM 3: RVtip to RVring (AP: atrial pace; PVC: premature ventricular contraction; VSP: ventricular safe pace; VP:ventricular pace) 


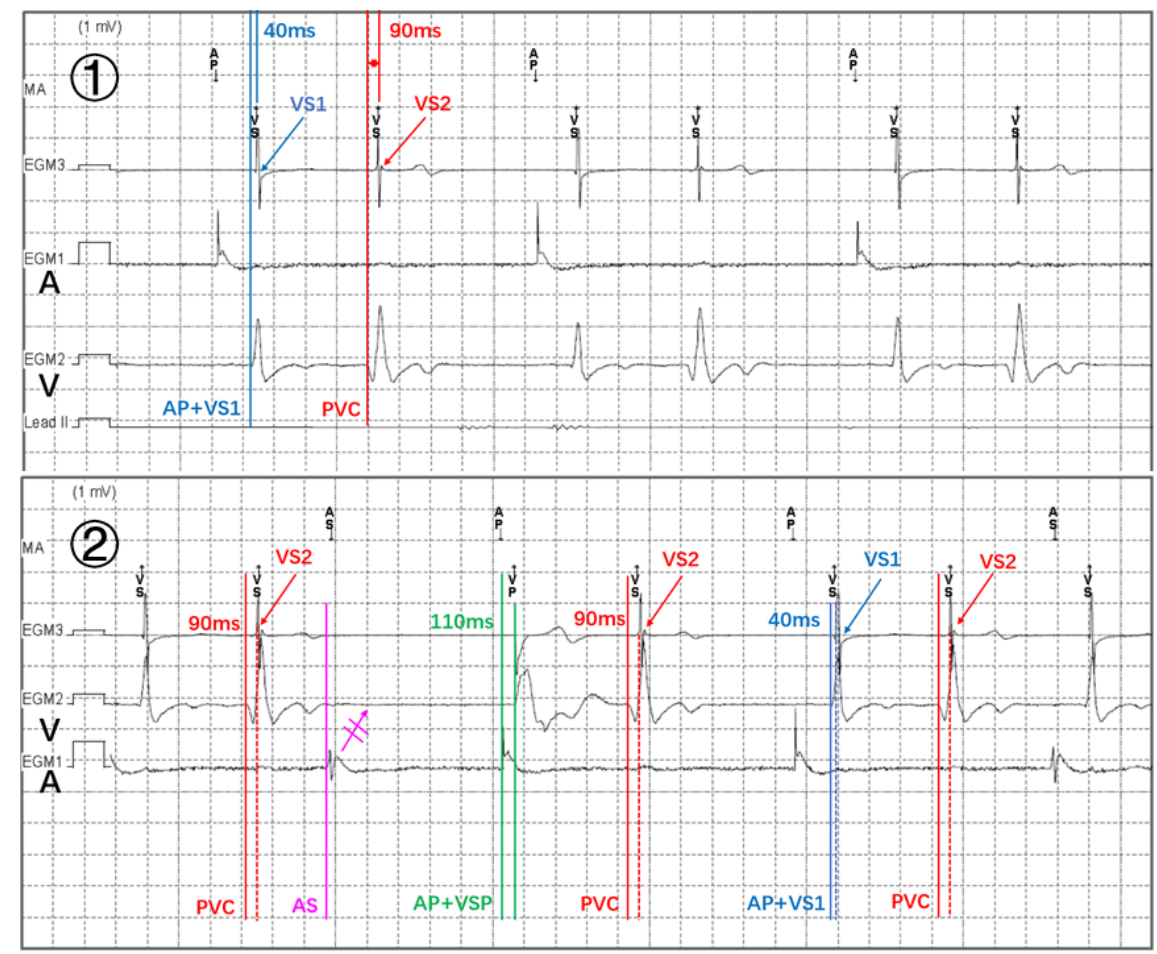

Figure-2: Pacing rate was adjusted from 60 to 55 times / min,and MVP on. MVP on and Beta-blocker dose increase. EGM 1:Atip to Aring;EGM 2:Can to RV coil;EGM 3: RVtip to RVring. (AP: atrial pace; PVC: premature ventricular contraction;VP:ventricular pace;VS:ventricular sense;VSP:ventricular safe pace;VS1:ventricular sense of ventricular activation transmitted through the atrioventricular node; VS2: ventricular sense of PVC originated from the scar zone of posterior inferior left ventricle)

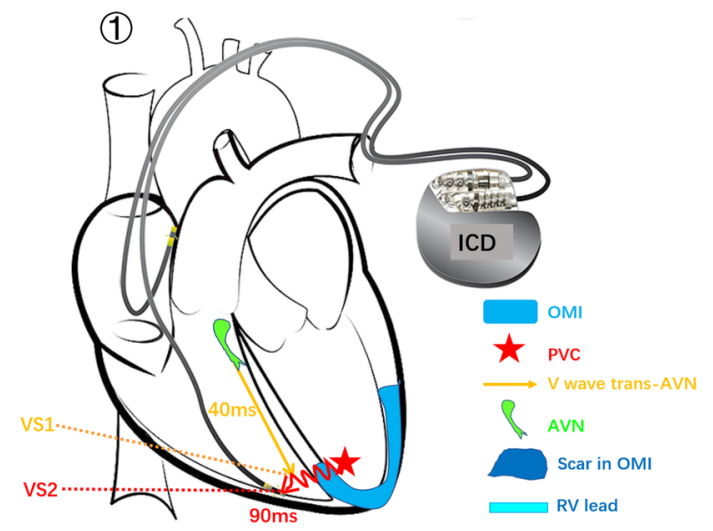

(2)

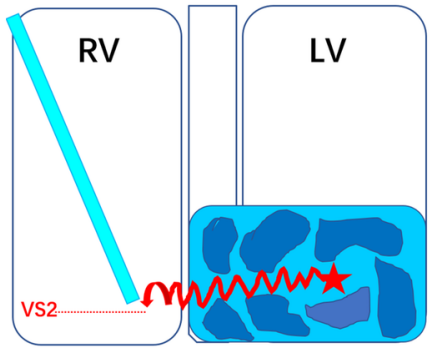

Figure-3: The main mechanism of the "troubleshooting": the slow conduction of the scar zone of posterior inferior left ventricle and interventricular septum due to the old myocardial infarction. EGM 1:Atip to Aring;EGM 2:Can to RV coil;EGM 3: RVtip to RVring . (AVN:atrioventricular node;OMI:old myocardial infarction;PVC: premature ventricular contraction;VS1:ventricular sense of ventricular activation transmitted 
through the AVN;VS2:ventricular sense of PVC originated from the scar of posterior inferior left ventricle) 